\title{
ON THE WHITEHEAD HOMOMORPHISM $J$
}

\author{
BY JOHN MILNOR ${ }^{1}$
}

Communicated by R. H. Fox, February 3, 1958

Consider the homomorphism $J: \pi_{r-1}\left(S O_{n}\right) \rightarrow \pi_{n+r-1}\left(S^{n}\right)$ of G. W. Whitehead ${ }^{2}$ in the stable range $n>r$. The object of this note is to prove:

THEOREM 2. Let $q$ be an odd prime and let $r$ be any multiple of $2(q-1) q^{i}, i \geqq 0$. Then for $n>r$ the image $J \pi_{r-1}\left(S O_{n}\right) \subset \pi_{n+r-1}\left(S^{n}\right)$ contains a cyclic subgroup of order $q^{i+1}$.

According to recent work of Adams (as yet unpublished) the stable group $\pi_{n+r-1}\left(S^{n}\right)$ has the following $q$-primary components:

$Z_{q}$ for $r=2 i(q-1), i<q$ (this result is due to Cartan);

$Z_{q}$ for $r=2 q(q-1)-1$;

$Z_{q^{2}}$ for $r=2 q(q-1)$; and zero for other values of $r$ less than $2 q(q-1)$. Comparing this with Theorem 2 we have:

CoRollary. For $r<2 q(q-1)-1$, and for $r=2 q(q-1)$, the image $J_{\pi_{r-1}}\left(S_{n}\right)$ contains the $q$-primary component of the stable group $\pi_{n+r-1}\left(S^{n}\right)$.

The corresponding assertion for $r=2 q(q-1)-1$ is false, since the group $\pi_{r-1}\left(S O_{n}\right)$ is zero ${ }^{3}$ in this case.

The proof will be based on work of Thom, Hirzebruch, Borel and von Staudt.

THEOREM 1. Let $\xi$ be the $S O_{n}$-bundle over $S^{r}$ corresponding ${ }^{4}$ to an element $\lambda$ of $\pi_{r-1}\left(S O_{n}\right)$. If $\lambda \lambda=0$ then there exists an oriented manifold $M^{r}$, differentiably imbedded in the sphere $S^{n+r}$, and having the following property: Some map $g: M^{r} \rightarrow S^{r}$ of degree +1 is covered by a bundle map of the normal bundle of $M^{r}$ into the given bundle $\xi$.

(It is not asserted that $M^{r}$ is connected.) The manifolds $M^{r}$ constructed in this way will be further studied in a later paper. ${ }^{5}$

Proof of Theorem 1 . Let $E$ be the total space of the $n$-cell bundle over $S^{r}$ associated with $\xi$; so that the boundary $\dot{E}$ is the total space

1 The author holds a Sloan fellowship.

2 G. W. Whitehead, Ann. of Math. vol. 43 (1942), pp. 634-640.

${ }^{3}$ See R. Bott, Proc. Nat. Acad. Sci. U.S.A. vol. 43 (1957) pp. 933-935.

${ }^{4}$ See Steenrod, The topology of fibre bundles, 1951, p. 99.

$5 \mathrm{~J}$. Milnor, A generalization of a theorem of Rohlin, to appear. 
of the associated $(n-1)$-sphere bundle. Consider the identification space $E / \dot{E}$ obtained by collapsing $\dot{E}$ to a point $e^{0}$. This space has a cell subdivision with cells $e^{0}, e^{n}$, and $e^{n+r}$; where $e^{n}$ corresponds to the inverse image of a base point in $S^{r}$. The Thom isomorphism ${ }^{6}$ $\Phi: H^{i}\left(S^{r}\right) \rightarrow H^{i+n}(E, \dot{E})$ can be used to give specific orientations to the cells $e^{n}, e^{n+r}$. In any such cell complex the cell $e^{n+r}$ is attached to the sphere $e^{0} \cup e^{n}$ by means of an attaching map $S^{n+r-1} \rightarrow e^{0} \cup e^{n}$ which is well defined up to homotopy.

LEMMA 1. The homotopy class of the attaching map, considered as an element of $\pi_{n+r-1}\left(S^{n}\right)$, is equal to $J \lambda$.

The proof, which is not difficult, will be given in a subsequent paper. $^{7}$

Now suppose that $\lambda \lambda=0$. Then the complex $E / \dot{E}$ has the homotopy type of the union $S^{n} \bigvee S^{n+r}$ with a single point in common; hence there exists a map $f: S^{n+r} \rightarrow E / \dot{E}$ of degree +1 .

The complement $E / \dot{E}-e^{0}$ can be considered as a differentiable manifold, with submanifold $S^{r}$ corresponding to the trivial crosssection of the cell bundle. Following Thom ${ }^{8}$ the map $f$ can be approximated by a map $f_{1}$ which is $t$-regular on $S^{r}$. The inverse image $M^{r}=f_{1}^{-1}\left(S^{r}\right)$ is then a differentiable manifold with a canonical orientation. Furthermore, if $g: M^{r} \rightarrow S^{r}$ denotes the restriction of $f_{1}$, then $g$ is covered by a bundle map of the normal bundle of $M^{r}$ into the given bundle $\xi$.

Let $T_{1}$ and $T_{2}$ be suitably chosen open tubular neighborhoods of $M^{r}$ and $S^{r}$. From the commutativity of the diagram

$$
\begin{array}{ccc}
B^{r}\left(S^{r}\right) & \stackrel{\Phi}{\rightarrow} H^{n+r}\left(E / \dot{E}, E / \dot{E}-T_{2}\right) \rightarrow H^{n+r}(E / \dot{E}) \\
\downarrow g^{*} & \downarrow & \downarrow f_{1}^{*} \\
H^{r}\left(M^{r}\right) & \stackrel{\Phi}{\rightarrow} B^{n+r}\left(S^{n+r}, S^{n+r}-T_{1}\right) \rightarrow B^{n+r}\left(S^{n+r}\right)
\end{array}
$$

it follows that $g$ has degree +1 . This completes the proof of Theorem 1.

Now suppose that $r$ is equal to $4 k$. The Pontrjagin classes $p_{i}$ of the manifold $M^{r}$ are clearly zero for $i<k$. Furthermore the Pontrjagin number $p_{k}\left[M^{r}\right]$ is equal to $-\left\langle p_{k}(\xi), \mu\right\rangle$, where $\mu$ denotes the standard generator of $H_{r}\left(S^{r} ; Z\right)$. For such a manifold the Hirzebruch index

- R. Thom, Ann. Sci. Ecole Norm. Sup. vol. 69 (1952) pp. 109-181.

$7 \mathrm{~J}$. Milnor, On spaces with a gap in cohomology, Theorem 3, Corollary 1, to appear.

${ }^{8}$ R. Thom, Comment. Math. Helv. vol. 28 (1954) pp. 17-86. 
formula ${ }^{9}$ reduces to $\tau\left(M^{r}\right)=s_{k} p_{k}\left[M^{r}\right]$; where $s_{1}=1 / 3, s_{2}=7 / 45, \cdots$, and in general $s_{k}$ equals $2^{2 k}\left(2^{2 k-1}-1\right) /(2 k)$ ! times the Bernoulli number $B_{k}$. Since $\tau\left(M^{r}\right)$ is an integer by definition this implies:

CoROllary 1. Let $\xi$ and $\lambda$ be as above, with $r=4 k$. The condition $J \lambda=0$ implies that $s_{k}\left\langle p_{k}(\xi), \mu\right\rangle$ is an integer.

Borel and Hirzebruch ${ }^{10}$ have constructed an example of an $\mathrm{SO}_{n}$ bundle $\xi_{0}$ over $S^{4 k}, n>4 k$, such that the number $\left\langle p_{k}\left(\xi_{0}\right), \mu\right\rangle$ is equal to $2(2 k-1)$ !. Let $\lambda_{0}$ be the corresponding element of $\pi_{4 k-1}\left(S O_{n}\right)$ and let $h$ be the order of its image $\lambda_{0}$. ( $h$ is a positive integer since the stable homotopy groups of spheres are known to be finite groups.) Then for the bundle $\xi_{1}$ corresponding to $h \lambda_{0}$ the number

$$
s_{k}\left\langle p_{k}\left(\xi_{1}\right), \mu\right\rangle=s_{k} h 2(2 k-1) !
$$

must be an integer. An immediate consequence is the following.

CoRollary 2. The order $h$ of the element $J \lambda_{0}$ of $\pi_{n+4 k-1}\left(S^{n}\right)$ is a multiple of the denominator of the rational number $2(2 k-1) ! s_{k}$, expressed as a fraction in lowest terms.

The author is indebted to Hirzebruch for calling his attention to the following two theorems, which will be used to compute the above denominator. An odd prime $q$ is said to be "of rank $k$ " if $2 k \equiv 0$ (modulo $q-1$ ). Let $b_{k}$ denote the product of all odd primes of rank $k$.

First theorem of von Staudt. ${ }^{11}$ The denominator of the Bernoulli number $B_{k}$, expressed as a fraction in lowest terms, is equal to $2 b_{k}$.

Thus, setting $B_{k}=a_{k} / 2 b_{k}$, the integer $a_{k}$ is odd and has no prime factor of rank $k$. Any positive integer $k$ can be expressed as a product $k=2^{t} k_{1} k_{2}$ with $k_{1} k_{2}$ odd, where all of the prime factors of $k_{1}$ are of rank $k$, while none of the prime factors of $k_{2}$ is of rank $k$.

Second theorem of von Staudt. ${ }^{11}$ The numerator $a_{k}$ of $B_{k}$ is congruent to zero modulo $k_{2}$.

Now the number $2(2 k-1) ! s_{k}=2^{2 k}\left(2^{2 k}-2\right) B_{k} / 2 k$ can be written as

$$
2^{2 k-t-2}\left(2^{2 k}-2\right)\left(a_{k} / k_{2}\right) / b_{k} k_{1} \text {. }
$$

The numerator and denominator of this expression are clearly integers. Furthermore the prime factors of the denominator are all odd primes of rank $k$. But no such prime divides the numerator. (The

${ }^{9} \mathrm{~F}$. Hirzebruch, Neue topologische Methoden in der algebraischen Geometrie, 1956, p. 85 .

${ }^{10} \mathrm{~A}$. Borel and F. Hirzebruch, Characteristic classes and homogeneous spaces, to appear.

${ }^{11}$ See for example N. Nielsen, Traité élémentaire des nombres de Bernoulli, 1923, pp. 240-250. 
condition $2 k \equiv 0(\bmod q-1)$ implies that $\left.2^{2 k}-2 \equiv-1(\bmod q).\right)$ Thus we have proved:

Lemma 2. The number $2(2 k-1) ! s_{k}$, when expressed as a fraction in lowest terms, has denominator $b_{k} k_{1}$.

Proof OF ThEOREM 2. If $r=4 k$ is a multiple of $2(q-1) q^{i}$, it follows that $q$ is of rank $k$. Hence $q$ divides $b_{k}$ and $q^{i}$ divides $k_{1}$; so that $q^{i+1}$ divides the denominator of $b_{k} k_{1}$. Together with Theorem 1, Corollary 2 this completes the proof.

\section{THE CARTESIAN PRODUCT OF A CERTAIN NONMANIFOLD AND A LINE IS $E^{4}$}

BY R. H. BING ${ }^{1}$

Communicated February 25, 1958

An upper semicontinuous decomposition $G$ of $E^{3}$ into points and tame arcs is defined in [1] such that the decomposition space $B$ is topologically different from $E^{3}$. Interesting properties of this space have also been given by Fort [4], Curtis $[2 ; 3]$, and Wilder [3]. We show that the cartesian product of the space $B$ and a line $E^{1}$ is topologically $E^{4}$. Perhaps the argument used is related to that employed by Arnold Shapiro to show that the cartesian product of a manifold described by Whitehead in [5] and a line is topologically $E^{4}$.

The arcs of the decomposition $G$ are intersections of double tori as shown in the figure. The solid double torus contains four double tori $T_{1}, T_{2}, T_{3}, T_{4}$ as shown; each $T_{i}$ in turn contains four double tori $T_{i 1}, T_{i 2}, T_{i 3}, T_{i 4}$ (not shown) imbedded in $T_{i}$ as $T_{1}, T_{2}, T_{3}, T_{4}$ were imbedded in $T$; more double tori are imbedded in the $T_{i j}$ 's; etc. The tame arcs of the decomposition $G$ are the components of

$$
T \cdot \Sigma T_{i} \cdot \Sigma T_{i j} \cdot \Sigma T_{i j k} \cdot \ldots \text {. }
$$

Although these tame arcs are mutually exclusive, it is not possible to get a 2 -sphere in $E^{3}$ that misses their sum and separates two of them. No topological cube in $T$ contains $T_{1}+T_{2}+T_{3}+T_{4}$.

When the cartesian product is taken, the extra dimension enables one to unravel certain linking handles in the sense that if $[a, b]$ is

${ }^{1}$ Work supported by the National Science Foundation under NSF Grant G3248 at the Institute for Advanced Study, Princeton, New Jersey. 